\title{
Character Association and Path Coefficient Analysis for Qualitative and Quantitative Traits in Green Chilli Genotypes (Capsicum annuum L.)
}

\author{
Sujata Kumari", R.S. Jawadagi, Revanappa, P.S. Ajjappalavara, \\ D.A. Peerjade and A.M. Nadaf \\ Department of vegetable Science, Bagalkot, College of Horticulture, University of Horticultural \\ Sciences Bagalkot, 587-104, Karnataka, India \\ *Corresponding author
}

\section{A B S T R A C T}

Keywords

Chilli, Correlation coefficient, Path coefficient analysis and Yield.

\section{Article Info}

Accepted:

21 September 2017

Available Online:

10 October 2017
15 chilli genotypes were grown under randomized block design with two replication during Kharif season, 2016-17 to study the genetic correlation and path coefficient analysis for 15 different quantitative and qualitative traits. The correlation study revealed that plant height at 90 DAT, plant height at 120 DAT, secondary branches at 90 DAT, fruit weight, stalk length and fruits per plant had significant and positive correlation at genotypic as well as phenotypic level suggesting the direct selection of these traits as less prone to environmental factors. The path coefficient analysis had direct positive via yield through number of fruits per plant, fruit weight, number of secondary branches at 90 DAT and ascorbic acid content. Selection for these traits would be more beneficial and rewarding in concentrating the economical yield.

\section{Introduction}

Chilli (Capsicum annuum L.) with chromosome number $2 \mathrm{n}=2 \mathrm{x}=24$ belongs to Solanaceae family which originated and bred in American Tropics of new world. Its domestication as one of the first cultivated crops in the America dated back to more than 6000 years ago based on archeological evidence in South Western Equador.

It derived from Greek word "Kapsimo" meaning to bite (Basu and De, 2003).The genus Capsicum comprised of 25 wild species and five as cultivated species which has been extensively cultivated as vegetable and spice since antiquity.
It is an indispensable crop used as essential ingredient in a variety of cuisines to annex tang and taste to the otherwise insipid food by providing colour and flavour. It possess high nutritive value and an excellent source of vitamin $\mathrm{C}$ and $\mathrm{A}$ along with minerals like folate, potassium, thiamine, molybdenum and manganese. Antioxidant properties in chilli are due to $\beta$-carotinoids and vitamin $\mathrm{A}$ and $\mathrm{C}$ (Simonne et al., 1997). Nonetheless in India, chilli is an important commercial crop, cultivated for vegetable, spice, and value added product. Chilli has been acknowledged for extreme hotness or pungency due to presence of capsaicinoids in which 
predominant forms are capsaicin (8- methylN-vanillyl-6-enamide) and dihydrocapsaicin making upto 80-90 per cent (Hoffman et al., 1983). The capsaicinoids have pharmaceutical applications namely, antioxidant, anti-obesity treatments, anti-arthritic, analgesic, antimicrobial and anticancerous properties (Prasad et al., 2005). Besides this, the colour exhibited in Capsicum is due to group of carotenoids of capsanthin, capsorubin, cryptoxanthine, zeaxanthine and others. The extracted colours of chilli are used in the food processing industries extensively to wide range of food products (Govindarajan, 1986).

India is the major producer as well as exporter of chilli. Major chilli growing states are Andhra Pradesh, Maharashtra, Tamil Nadu, Karnataka, Orissa, Uttar Pradesh and Bihar. Among all the vegetables, chilli alone occupies an area of 238('000) ha with the production of 2392('000) MT (Anonymous, 15-16).

Yield is an intricate quantitative character governed by large number of genes and magnanimously influenced by environmental factors. The advancement of breeding in a population primarily determined by the nature, magnitude and interaction of genotypic and environmental variations. Thus, after analysing the relationship between two or more variables, correlation coefficient determines the component character on which selection can be done whereas path coefficient analysis partition the correlation coefficients into direct and indirect effects for easy selection procedure.

\section{Materials and Methods}

The field experiment was conducted at Horticulture Research and Extension Station, Vijayapur (Tidagundi), University of Horticultural Sciences, Bagalkot during Kharif 2016- 17. The experiment was laid out in Randomized block design with two replication. Fifteen germplasms (Table 1) were studied with the spacing of $75 \mathrm{~cm} \times$ $45 \mathrm{~cm}$. The University prescribed package of practices were adopted for growing Chilli.

Observations were noted down on five randomly selected plants in each replication for fifteen quantitative and qualitative traits viz., Plant height $(\mathrm{cm})$ at 90 DAT, Plant height $(\mathrm{cm})$ at 120 DAT, Primary branches at 90 DAT, Secondary branches at 90 DAT, Days to first flowering, Days to fifty percent flowering, Fruit length $(\mathrm{cm})$, Fruit weight $(\mathrm{gm})$, Fruit width $(\mathrm{cm})$, Stalk length $(\mathrm{cm})$, Fruits per plant, Ascorbic acid $\left({ }^{0} \mathrm{~B}\right)$, Total soluble solids, Capsaicin percent and Fruit yield per plant (t/ha). The data were further subjected for analysis to calculate genotypic and phenotypic correlation coefficient as per Al- Jibourie et al., (1958). Dewey and Lu (1959) method was employed for calculating direct and indirect effects of component characters on yield.

\section{Results and Discussion}

\section{Correlation coefficient analysis}

Correlation coefficient was worked out to assist in selection procedure which suggest the association of traits with yield. The association among the component traits listed in Tables 2 and 3. Plant height at 90 DAT (0.952, 0.630), Plant height at 120 DAT (0.899, 0.556), Secondary branches at 90 DAT $(0.985,0.553)$, fruit weight $(0.497$, $0.490)$, stalk length $(0.686,0.488)$ and fruits per plant $(0.989,0.778)$ had significant and positive correlation with green Chilli yield per plant at genotypic and phenotypic level respectively. This is in tune with the research work conducted by Patel and Patel (2014), Yatung et al., (2014) and Dolkar et al., (2015), Patel et al., (2015), Sharma and Sridevi (2016), and Mamatha et al., (2016). 
High positive significant correlation was also recorded for days to fifty percent flowering $(0.387,0.240)$ which is in conformity with Kumari et al., (2010) and Pandit and Adhikary (2014).

In consideration to fruit quality parameters, capsaicin content only had positive and significant association with yield (0.513, 0.413). Earlier Kumari et al., (2010), Patel et al., (2015), and Srividhya et al., (2017) also penned down the positive association with fruit yield.

\section{Path coefficient analysis}

In the present study (Tables 4 and 5), for selected 15 characters, the traits such as number of fruits per plant $(0.672,0.865)$, fruit weight $(0.295,0.385)$, stalk length (0.294, 116) had strong direct positive effect on yield which is in agreement with Yatung et al., (2014), Pandit and Adhikary (2014), Patel et al., (2015), Mamatha et al., (2016) and Sharma and Sridevi (2016) at genotypic as well as phenotypic level. The biochemical parameter viz., ascorbic acid also has direct positive effect towards yield $(0.223,0.296)$ which is in consonance with Chattopadhyay et al., (2011), Vikram et al., (2014) and Patel et al., (2015). Thus, the direct selection for these traits would be rewarding for improvement of economical yield. Some of the traits such as plant height at 90 DAT, days to first flowering, fruit width and capsaicin percent had very low to negligible positive direct effect which indicates the association is less strong.

Table.1 Details of chilli genotypes used in experiment and their source of collections

\begin{tabular}{|c|c|c|}
\hline Treatment & Genotypes & Source \\
\hline $\mathrm{T} 1$ & DC-1601 & Devihosur \\
\hline $\mathrm{T} 2$ & DC-1602 & Devihosur \\
\hline T3 & DC-1603 & Devihosur \\
\hline $\mathrm{T} 4$ & DC-1604 & Devihosur \\
\hline T5 & DC-1605 & Devihosur \\
\hline T6 & DC-1606 & Devihosur \\
\hline $\mathrm{T} 7$ & DC-1608 & Devihosur \\
\hline $\mathrm{T} 8$ & DC-1609 & Devihosur \\
\hline T9 & DC-1610 & Devihosur \\
\hline T10 & DC-1611 & Devihosur \\
\hline T11 & DC-1612 & Devihosur \\
\hline T12 & DC-1613 & Devihosur \\
\hline T13 & DC-1614 & Devihosur \\
\hline $\mathrm{T} 14$ & DC-1615 & Devihosur \\
\hline $\mathrm{T} 15$ & DC-1007 & UAS, Dharwad \\
\hline
\end{tabular}


Table.2 Genotypic correlation coefficients among quantitative and qualitative parameters in green chilli

\begin{tabular}{|c|c|c|c|c|c|c|c|c|c|c|c|c|c|c|c|}
\hline & 1 & 2 & 3 & 4 & 5 & 6 & 7 & 8 & 9 & 10 & 11 & 12 & 13 & 14 & 15 \\
\hline 1 & 1.00 & $0.975^{* *}$ & 0.197 & $0.741 * *$ & 0.001 & 0.319 & 0.268 & $0.507^{* *}$ & -0.322 & $0.922 * *$ & $0.968 * *$ & -0.174 & 0.019 & $0.504 * *$ & $0.952 * *$ \\
\hline 2 & & 1.00 & 0.262 & $0.765 * *$ & 0.233 & $0.376^{*}$ & 0.280 & $0.433^{*}$ & -0.107 & 0.939 ** & $0.783 * *$ & 0.014 & 0.107 & $0.431 *$ & $0.899 * *$ \\
\hline 3 & & & 1.00 & -0.221 & 0.119 & -0.124 & -0.253 & 0.204 & $0.562^{* *}$ & 0.130 & 0.182 & -0.144 & -0.234 & $-0.423 *$ & 0.236 \\
\hline 4 & & & & 1.00 & 0.220 & 0.166 & $0.375^{*}$ & $0.549 * *$ & $-0.369^{*}$ & $0.807 * *$ & $0.849^{* *}$ & -0.095 & -0.182 & $0.618 * *$ & $0.985^{* *}$ \\
\hline 5 & & & & & 1.00 & $0.419^{*}$ & -0.163 & -0.134 & -0.055 & 0.025 & 0.160 & 0.247 & 0.267 & 0.008 & 0.344 \\
\hline 6 & & & & & & 1.00 & 0.216 & $0.549^{* *}$ & -0.224 & $0.376^{*}$ & 0.053 & $0.363 *$ & -0.097 & $0.396^{*}$ & $0.387^{*}$ \\
\hline 7 & & & & & & & 1.00 & 0.243 & $-0.506^{* *}$ & $0.539 * *$ & 0.003 & 0.254 & $-0.424 *$ & -0.083 & 0.027 \\
\hline 8 & & & & & & & & 1.00 & $0.411^{*}$ & 0.262 & 0.284 & -0.209 & -0.296 & $0.479 * *$ & 0.497 ** \\
\hline 9 & & & & & & & & & 1.00 & -0.277 & -0.332 & 0.037 & -0.284 & 0.0000 & -0.100 \\
\hline 10 & & & & & & & & & & 1.00 & $0.568 * *$ & -0.069 & $-0.424 *$ & 0.042 & $0.686^{* *}$ \\
\hline 11 & & & & & & & & & & & 1.00 & -0.326 & 0.156 & $0.494 * *$ & $0.989 * *$ \\
\hline 12 & & & & & & & & & & & & 1.00 & -0.015 & -0.123 & -0.185 \\
\hline 13 & & & & & & & & & & & & & 1.00 & 0.194 & -0.137 \\
\hline 14 & & & & & & & & & & & & & & 1.00 & $0.513 * *$ \\
\hline
\end{tabular}

$*$ Significant at $\mathrm{p}=0.05$, Critical $\mathrm{r}_{\mathrm{g}}$ value at $1 \%=0.46, * *$ Significant at $\mathrm{p}=0.01$
1. Plant height at 90 DAT
5. Days to first flowering
9. Fruit width
6. Days to 50 per cent flowering
10. Stalk length
2. Plant height at $120 \mathrm{DAT}$
3. Primary branches at $90 \mathrm{DAT}$
7. Fruit length
11. Fruits per plant
4. Secondary branches at 90 DAT
8. Fruit weight
12. Ascorbic acid

13. Total soluble solids

15. yield per pla

Table.3 Phenotypic correlation coefficients among quantitative and qualitative parameters in green chilli

\begin{tabular}{|c|c|c|c|c|c|c|c|c|c|c|c|c|c|c|c|}
\hline & 1 & 2 & 3 & 4 & 5 & 6 & 7 & 8 & 9 & 10 & 11 & 12 & 13 & 14 & 15 \\
\hline 1 & 1.00 & $0.795 * *$ & 0.097 & $0.579^{* * *}$ & 0.082 & 0.137 & 0.186 & 0.247 & -0.111 & $0.635^{* * *}$ & $0717 * *$ & 0.144 & 0.152 & $0.408^{*}$ & $0.630^{* * *}$ \\
\hline 2 & & 1.00 & 0.076 & $0.474 * *$ & 0.251 & 0.135 & 0.221 & 0.255 & -0.027 & $0.669^{* * *}$ & $0578^{* *}$ & 0.011 & 0.124 & 0.341 & $0.556^{* *}$ \\
\hline 3 & & & 1.00 & -0.129 & 0.137 & -0.016 & -0.089 & 0.216 & $0.413^{*}$ & 0.075 & 0.056 & 0.021 & -0.322 & -0.224 & 0.175 \\
\hline 4 & & & & 1.00 & 0.224 & 0.248 & 0.143 & 0.262 & -0.099 & 0.355 & $0.646^{* * *}$ & 0.151 & -0.039 & $0.449 * *$ & $0.553^{* *}$ \\
\hline 5 & & & & & 1.00 & 0.193 & -0.121 & -0.122 & 0.044 & 0.007 & 0.114 & 0.183 & 0.188 & 0.016 & 0.209 \\
\hline 6 & & & & & & 1.00 & 0.049 & 0.344 & 0.136 & 0.053 & 0.082 & 0.227 & 0.035 & 0.184 & 0.240 \\
\hline 7 & & & & & & & 1.00 & 0.243 & $-0.477 * *$ & $0.499 * *$ & 0.022 & 0.287 & -0.352 & -0.091 & 0.052 \\
\hline 8 & & & & & & & & 1.00 & 0.318 & 0.217 & 0.213 & -0.153 & -0.292 & $0.394 *$ & $0.490^{* *}$ \\
\hline 9 & & & & & & & & & 1.00 & -0.261 & -0.324 & 0.055 & -0.147 & 0.052 & -0.025 \\
\hline 10 & & & & & & & & & & 1.00 & $0.519^{* * *}$ & 0.093 & -0.266 & 0.047 & $0.488 * *$ \\
\hline 11 & & & & & & & & & & & 1.00 & $-0.372^{*}$ & 0.100 & $0.388^{*}$ & $0.778^{* *}$ \\
\hline 12 & & & & & & & & & & & & 1.00 & -0.025 & -0.126 & -0.096 \\
\hline 13 & & & & & & & & & & & & & 1.00 & 0.109 & -0.071 \\
\hline 14 & & & & & & & & & & & & & & 1.00 & $0.413^{*}$ \\
\hline 15 & & & & & & & & & & & & & & & 1.00 \\
\hline
\end{tabular}

$*$ Significant at $\mathrm{p}=0.05$, Critical $\mathrm{r}_{\mathrm{g}}$ value at $1 \%=0.46, * *$ Significant at $\mathrm{p}=0.01$
1. Plant height at 90 DAT
5. Days to first flowering
2. Plant height at 120 DAT
6. Days to 50 per cent flowering
7. Fruit length
3. Primary branches at 90 DAT
8. Fruit weight

9. Fruit width

10. Stalk length

13. Total soluble solids

11. Fruits per plant

14. Capsaicin percent

15. yield per plant

12. Ascorbic acid 
Table.4 Genotypic path coefficient analysis among quantitative and qualitative parameters in green chilli

\begin{tabular}{|c|c|c|c|c|c|c|c|c|c|c|c|c|c|c|c|}
\hline & 1 & 2 & 3 & 4 & 5 & 6 & 7 & 8 & 9 & 10 & 11 & 12 & 13 & 14 & rG \\
\hline 1 & 0.125 & -0.445 & 0.034 & 0.199 & 0.000 & 0.051 & -0.125 & 0.149 & 0.083 & 0.271 & 0.650 & -0.039 & -0.004 & 0.002 & $0.952 * *$ \\
\hline 2 & 0.14 & -0.397 & 0.045 & 0.205 & 0.035 & 0.060 & -0.13 & 0.128 & 0.028 & 0.276 & 0.526 & 0.003 & -0.021 & 0.001 & 0.899 \\
\hline 3 & 0.025 & -0.104 & 0.171 & -0.059 & 0.018 & -0.019 & 0.117 & 0.060 & -0.145 & 0.038 & 0.122 & -0.032 & 0.045 & -0.001 & 0.236 \\
\hline 4 & 0.093 & -0.303 & -0.039 & 0.268 & 0.033 & 0.027 & -0.174 & 0.162 & 0.095 & 0.238 & 0.569 & -0.021 & 0.035 & 0.002 & $0.985^{* *}$ \\
\hline 5 & 0.000 & -0.092 & 0.020 & 0.059 & 0.148 & 0.067 & 0.076 & -0.039 & -0.014 & 0.007 & 0.108 & 0.055 & -0.051 & 0 & 0.344 \\
\hline 6 & 0.039 & -0.149 & -0.021 & 0.044 & 0.062 & 0.161 & -0.1001 & 0.162 & -0.058 & 0.111 & 0.036 & 0.081 & 0.019 & 0.001 & $0.387 *$ \\
\hline 7 & 0.034 & -0.111 & -0.043 & 0.101 & -0.024 & 0.035 & -0.464 & 0.072 & 0.131 & 0.159 & 0.002 & 0.057 & 0.081 & -0.000 & 0.027 \\
\hline 8 & 0.063 & -0.172 & 0.035 & 0.147 & -0.019 & 0.088 & -0.113 & 0.295 & -0.106 & 0.077 & 0.191 & -0.047 & 0.057 & 0.002 & $0.497 * *$ \\
\hline 9 & -0.040 & 0.042 & 0.096 & -0.099 & 0.008 & 0.036 & 0.235 & 0.121 & -0.258 & -0.081 & -0.223 & 0.008 & 0.055 & 0 & -0.100 \\
\hline 10 & 0.115 & -0.373 & 0.022 & 0.217 & 0.004 & 0.060 & -0.249 & 0.077 & 0.0713 & 0.294 & 0.381 & -0.016 & 0.081 & 0.000 & $0.686^{* *}$ \\
\hline 11 & 0.121 & -0.3 & 0.031 & 0.228 & 0.024 & 0.009 & -0.001 & 0.084 & 0.086 & 0.16 & 0.672 & -0.073 & -0.03 & 0.002 & $0.989 * *$ \\
\hline 12 & -0.022 & -0.006 & -0.025 & -0.026 & 0.037 & 0.058 & -0.118 & -0.062 & -0.009 & -0.021 & -0.219 & 0.223 & 0.003 & -0.000 & -0.185 \\
\hline 13 & 0.002 & -0.042 & -0.040 & -0.049 & 0.039 & -0.016 & 0.197 & -0.087 & 0.073 & -0.125 & 0.105 & -0.003 & -0.192 & 0.001 & -0.137 \\
\hline 14 & 0.063 & -0.171 & -0.073 & 0.166 & 0.001 & 0.064 & 0.039 & 0.141 & 0 & 0.012 & 0.332 & -0.027 & -0.037 & 0.003 & 0.513 \\
\hline
\end{tabular}

Residual effect= SQRT (1-1.2043) Bold diagonal figures indicate direct effect $\mathrm{rG}=$ Genotypic correlation coefficient of Total yield

$*$ and $* *$ indicate significant at 5 and 1 percent probability level, respectively
1. Plant height at 90 DAT
5. Days to first flowering
6. Days to 50 per cent flowering
7. Fruit length
3. Primary beanches at 90 DAT
8. Fruit weight

9. Fruit width

10. Stalk length
11. Fruits per plant

13. Total soluble solids

14. Capsaicin percent

12. Ascorbic acid

Table.5 Phenotypic path coefficient analysis among quantitative and qualitative parameters in green chilli

\begin{tabular}{|c|c|c|c|c|c|c|c|c|c|c|c|c|c|c|c|}
\hline & 1 & 2 & $\mathbf{3}$ & 4 & 5 & 6 & 7 & 8 & 9 & 10 & 11 & 12 & 13 & 14 & $\mathbf{r P}$ \\
\hline 1 & 0.142 & -0.137 & -0.011 & -0.069 & 0.015 & -0.007 & -0.017 & 0.096 & -0.015 & 0.073 & 0.620 & $\begin{array}{c}-0.043 \\
\end{array}$ & -0.014 & -0.005 & $0.630^{* *}$ \\
\hline 2 & 0.113 & -0.173 & -0.008 & -0.057 & 0.047 & -0.007 & -0.020 & 0.099 & -0.004 & 0.077 & 0.500 & 0.003 & -0.011 & -0.004 & $0.556^{* *}$ \\
\hline 3 & 0.014 & -0.013 & -0.109 & 0.016 & 0.026 & 0.001 & 0.008 & 0.084 & 0.055 & 0.009 & 0.048 & 0.006 & 0.029 & 0.003 & 0.175 \\
\hline 4 & 0.082 & -0.082 & 0.014 & -0.119 & 0.042 & -0.012 & -0.013 & 0.102 & -0.013 & 0.041 & 0.559 & -0.045 & 0.004 & -0.006 & $0.553^{* *}$ \\
\hline 5 & 0.012 & -0.043 & -0.015 & -0.027 & 0.186 & -0.009 & 0.011 & -0.047 & 0.006 & 0.001 & 0.099 & 0.054 & -0.017 & -0.000 & 0.209 \\
\hline 6 & 0.019 & -0.023 & 0.002 & -0.029 & 0.036 & -0.049 & -0.005 & 0.134 & 0.0181 & 0.006 & 0.071 & 0.067 & -0.003 & -0.002 & 0.240 \\
\hline 7 & 0.026 & -0.038 & 0.009 & -0.017 & -0.027 & -0.003 & -0.092 & 0.094 & -0.063 & 0.058 & -0.019 & 0.085 & 0.032 & 0.001 & 0.052 \\
\hline 8 & 0.035 & 0.044 & -0.024 & -0.031 & -0.023 & -0.017 & -0.022 & 0.389 & 0.042 & 0.025 & 0.184 & -0.045 & 0.027 & -0.005 & $0.490 * *$ \\
\hline 9 & -0.016 & 0.005 & -0.045 & 0.012 & 0.008 & -0.007 & 0.044 & 0.124 & 0.133 & -0.030 & -0.281 & 0.016 & 0.013 & -0.001 & -0.025 \\
\hline 10 & 0.090 & -0.116 & -0.008 & -0.043 & 0.001 & -0.003 & -0.047 & 0.084 & -0.035 & 0.116 & 0.449 & -0.028 & 0.024 & -0.001 & $0.488^{* *}$ \\
\hline 11 & 0.102 & -0.099 & -0.006 & -0.077 & 0.021 & -0.004 & 0.002 & 0.083 & -0.043 & 0.06 & 0.865 & -0.110 & -0.009 & -0.005 & $0.779 * *$ \\
\hline 12 & -0.021 & -0.002 & -0.002 & 0.018 & 0.034 & -0.011 & -0.026 & -0.059 & 0.007 & -0.011 & -0.323 & 0.296 & 0.002 & 0.002 & -0.096 \\
\hline 13 & 0.022 & -0.021 & 0.035 & 0.005 & 0.035 & -0.002 & 0.032 & -0.114 & -0.019 & -0.031 & 0.087 & -0.007 & -0.091 & -0.001 & -0.070 \\
\hline 14 & 0.058 & -0.059 & 0.025 & -0.054 & 0.003 & -0.009 & 0.008 & 0.153 & 0.007 & 0.006 & 0.335 & -0.037 & -0.009 & -0.013 & $0.413^{*}$ \\
\hline
\end{tabular}

Residual effect $=0.423$ Bold diagonal figures indicate direct effect. $\mathrm{rP}=$ Phenotypic correlation coefficient of total yield

$*$ and $* *$ indicate significant at 5 and 1 percent probability level, respectively.
1. Plant height at 90 DAT
5. Days to first flowering
9. Fruit width
10. Stalk length
11. Fruits per plant
7. Fruit length
12. Ascorbic acid
13. Total soluble solids
3. Primary branches at 90 DAT
8. Fruit weight
14. Capsaicin percent
15. yield per plant 
The negative direct effect via fruit yield per plant showed that plant height at 120 DAT (0.397), fruit length (-0.464) and fruit width (0.258 ) are in association with Vikram et al., (2014), Vijaya et al., (2014), Kadwey et al., (2015) and Abhinaya et al., (2016). The total soluble solids (-0.192) had indirect effect via yield which are in accordance with Sood et al., (2007). These negative direct effects are considered for nullifying the indirect effects on fruit yield per plant.

From the present investigation based on correlation and path coefficient analysis the characters such as plant height at 90 DAT, secondary branches, number of fruits per plant, fruit weight and stalk length can directly be employed in any selection procedure for yield improvement.

\section{References}

Abhinaya, M., Modha, K. G., Patel, R. K. and Parmar, H. B., 2016, Genetic diversity analysis for dry fruit yield, its attributes and quality traits in chilli (Capsicum annuum L.). Electron J. Plant Breed, 7 (4): 1200-1207.

Al-Jibourie, H. A., Miller, P. A. and Robinson, H. V., 1958, Genotypic and environmental variances and covariances in addition to upland cotton cross of interspecific origin. Agron. J., 50: 533-536.

Anonymous, 2016, Indian Horticultural Database- 2016. http: //www. nhb. gov. in.

Basu, S. K., and De, A. K., 2003, Capsicum: historical and botanical perspectives. In: De, A. K., editor. Capsicum: The Genus Capsicum. London: CRC Press; p. 1-15. Chattopadhyay, A., Sharangi, A. B., Dai, N. and Dutt, S., 2011, Diversity of genetic resources and genetic association analyses of green and dry chillies of Eastern India. Chilean J. Agric. Res., 71

\section{(3): 350-355.}

Dewey, D. R., and Lu, K. H., 1959, A correlation and path coefficient analysis of components of crested wheat grass seed production. Agron. J., 51: 515-518.

Dolkar, R., Madalageri, M. B. and Manjunath, G., 2015, Correlation and path analysis for growth, earliness, yield and quality parameters in Chilli (Capsicum annuum L.). Hort. Flora Res. Spectrum, 4 (3): 268-272.

Govindarajan, V. S., 1986, Capsicum production, technology, chemistry and quality. Part III. Chemistry of the color, aroma and pungency stimuli. Crit. Rev. Food Sci. Nutr., 24: 245-355.

Hoffman, P. G., Lego, M. C., and Galetto, W. G., 1983, Separation and quantitation of red pepper major heat principles by reverse-phase high pressure liquid chromatography. J. Agril. Food Chem., 31: 1326-1330.

Kadwey, S., Dadiga, A. Prajapati, S. and Telugu, R. K., 2015, Correlation and path co-efficient analysis of quantitative and qualitative traits in Chilli (Capsicum annuum L.). The Bioscan, 10 (4): 1909-1914.

Kumari, S. S., Jyothi, K. U., Srihari, D., Sankar, A. S. and Sankar, C. R., 2010, Character association in Paprika (Capsicum annuum L.). J. Spices and Aromatic Crops. 16: 71-75.

Mamatha, A., Devaraju, P. C. U. and Srinivasa, V., 2016, Genetic correlation and path coefficient analysis In Chilli (Capsicum annuum L.) genotypes under hill zone of Karnataka. The Bioscan, 11 (3):1995-1998.

Pandit, M. K., and Adhikary, S., 2014, Variability and heritability estimates in some reproductive characters and yield in Chilli (Capsicum annuum L.). International J. of Plant \& Soil Sci., 3 (7): 845-853.

Patel, D. K., and Patel, J. R., 2014, 
Correlation and path analysis in Chilli (Capsicum annuum var. Longum (DC.)Sendt.). Trends in Biosciences, 7 (24): 4422-4425.

Patel, D. K., Patel, B. R., Patel, J. R. and Kuchhadiya, G. V., 2015, Genetic variability and character association studies for green fruit yield and quality component traits in Chilli (Capsicum апnиит var. longum (dc.) sendt.). Electron. J. Plant Breed, 6 (2): 472-478.

Prasad, N. B. C., Shrivastava, R. and Ravishankar, G. A., 2005, Capsaicin: a promising multifaceted drug from Capsicum spp. Evidence-Based Integrative Medicine, 2:147-166.

Sharma, M., and Sridevi, O., 2016, Genetic variability, correlation and pathcoefficient analysis for yield and yield related traits in Chilli (Capsicum annuum L.). Adv. in Life Sci., 5 (1): 144-147.

Simonne, A. H., Simonne, E. H., Eitenmiller, R. R., Mills, H. A. and Green, N. R., 1997, Ascorbic acid and pro-vitamin A contents in unusually colored bell peppers (Capsicum annuum L.). J. Food Compo. Anal., 10: 299-311.
Sood, S., Bindal, A. and Sharma, A., 2007, Genetical study for quality traits in Bell pepper [Capsicum annuum (L.) var. grossum Sendt.]. Indian J. Genet., 67 (1): 95-96.

Srividhya, S., Kumar, S. R. and Yassin, G. M., 2017, Association analysis in Chilli genotypes for dry fruit yield. International J. Hort., 7 (7): 47-53.

Vijaya, H. M., Mallikarjuna Gowda, A. P. and Nehru, S. D., 2014, Genetic variability, correlation coefficient and path analysis in Chilli (Capsicum annuum L.) genotypes. Res. Environ. Life Sci., 7 (3): 175- 178.

Vikram, A., Warshamana, I. K. and Gupta, M., 2014, Genetic correlation and path coefficient studies on yield and biochemical traits in Chilli (Capsicum annuum L). International J. Farm Sci., 4 (2): 70-75.

Yatung, T., Dubey, R. K., Singh, V., Upadhayay, G. and Pandey, A. K., 2014, Selection parameters for fruit yield and related traits in Chilli (Capsicum annuum L.). Bangladesh J. Bot., 43 (3): 283-291.

\section{How to cite this article:}

Sujata Kumari, R.S. Jawadagi, Revanappa, P.S. Ajjappalavara, D.A. Peerjade and Nadaf, A.M. 2017. Character Association and Path Coefficient Analysis for Qualitative and Quantitative Traits in Green Chilli Genotypes (Capsicum annuиm L.). Int.J.Curr.Microbiol.App.Sci. 6(10): 2273-2279. doi: https://doi.org/10.20546/ijcmas.2017.610.269 